\title{
Resposta imunológica a antígenos de Hysterothylacium deardorffoverstreetorum de peixes teleósteos
}

\author{
[Imunne response against Hysterothylacium deardorffoverestreetorum from teleost fish] \\ J. Ribeiro ${ }^{1}$, M. Knoff ${ }^{1}$, N.N. Felizardo ${ }^{1}$, M.A. Vericimo ${ }^{2}$, S.C. São Clemente ${ }^{3}$ \\ ${ }^{1}$ Aluno de pós-graduação - Faculdade de Medicina Veterinária - Universidade Federal Fluminense - Niterói, RJ \\ ${ }^{2}$ Instituto de Biologia - Universidade Federal Fluminense - Niterói, RJ \\ ${ }^{3}$ Faculdade de Medicina Veterinária - Universidade Federal Fluminense - Niterói, RJ
}

\begin{abstract}
RESUMO
Anisaquidose é uma doença provocada por parasitos da família Anisakidae e se caracteriza por manifestações gastrointestinais e alérgicas. O Anisakis simplex é o parasito mais patogênico ao homem e altamente alergênico. Porém, outros anisaquídeos também são danosos aos humanos, mas é desconhecida a imunogenicidade dessas larvas. $\mathrm{O}$ objetivo deste trabalho foi avaliar o potencial imunogênico do parasito Hysterothylacium deardorffoverestreetorum (HD) em modelo murino. Camundongos da linhagem $\mathrm{BALB} / \mathrm{c}$ foram divididos em três grupos experimentais e receberam as preparações antigênicas obtidas de larvas de HD: extrato bruto de larvas (EBH), extrato secretado/ excretado de larvas (ESH) e extrato bruto de larvas após excreção/secreção (EEH). Amostras séricas foram obtidas em diferentes dias após imunização para determinação dos níveis de anticorpos específicos pelo ensaio imunoenzimático (ELISA). Os resultados demonstram aumento na produção de imunoglobulina (Ig) $\mathrm{G}$ após a segunda imunização, com aumento progressivo após a terceira imunização. Já em relação à IgE, a reatividade foi mais tardia, demonstrando aumento progressivo após a terceira imunização. Foi avaliada a imunidade celular por meio da intradermorreação, como resultado estatisticamente significativo em relação ao controle utilizado. Este experimento é a primeira descrição da potencialidade patogênica desse parasito em mamíferos e representa um avanço no diagnóstico da anisaquidose humana.
\end{abstract}

Palavras-chave: anisaquidose, Hysterothylacium, infecção experimental, modelo murino

\begin{abstract}
Anisaquidosis is a disease caused by parasites of Anisakidae family and is characterized by gastrointestinal and allergic reactions. The Anisakis simplex is a more pathogenic Anisakidae to humans and is highly allergenic. However, other species of this family also have characteristics that are harmful to humans, but little is known about the immunogenicity this parasites. The objective of this study was to experimentally assess the immunogenic potential of the parasite Hysterothylacium deardorffoverestreetorum (H.D) in mice. Mice of inbred BALB/C strain were divided into three groups and received three immunizations of the following antigenic preparations obtained from L3 larvae H.D: Crude larval extract of H.D (CEH) Extract secreted / excreted larvae H.D. (ESH) and crude extract of larvae after excretion / secretion (EEH). Serum samples were obtained on different days after immunization to determine the levels of circulating specific antibodies by enzyme-linked immunosorbent assay (ELISA). The results show increased production of immunoglobulin (Ig) $G$ after the second immunization with a gradual increase after the third immunization. Regarding IgE reactivity, this occurred later, demonstrating a progressive increase only after the third immunization. Cellular immunity was evaluated by intradermal, and showed statistically significant result compared to the control used. This experiment is the first description of the pathogenic potential of this parasite in mammals and represents a breakthrough in the diagnosis of human Anisakidosis.
\end{abstract}

Keywords: anisakidosis, Hysterothylacium, experimental infection, murine model

Recebido em 26 de agosto de 2016

Aceito em 4 de outubro de 2016

E-mail: jana.ribeirovet@gmail.com 


\section{INTRODUÇÃO}

Os peixes teleósteos capturados e comercializados na costa brasileira são comumente parasitados por nematoides das famílias Anisakidae e Raphidascarididae. Parasitos membros dessas famílias utilizam os peixes como hospedeiros intermediários e podem ser encontrados em vísceras e musculatura (Cruz et al., 2010). O homem atua como hospedeiro acidental ao consumir o pescado cru, insuficientemente cozido, defumado a frio ou inadequadamente salgado, contendo larvas de terceiro estágio desses nematoides (Sabater; Sabater, 2000). Dessa forma, a ingestão acidental de larvas da família Anisakidae pode ocasionar uma doença conhecida como anisaquidose. Atualmente, sabe-se que nematoide Anisakis simplex é a espécie mais importante para a saúde pública, seguido de Pseudoterranova decipiens (Hochberg e Hamer, 2010). Larvas desses parasitos possuem um grande poder de sensibilização do sistema imunológico, o que pode resultar em manifestações alérgicas e gastrointestinais severas (Hochberg e Hamer, 2010; Cho et al., 2014). No entanto, com rara ocorrência, outros membros são citados como causadores de anisaquidose, como larvas de Contracaecum sp. e de Hysterothylacium aduncum. (Yagi et al., 1996; Barros et al., 2006; Hochberg e Hamer, 2010; Piscaglia et al., 2014). Com relação a estas duas últimas espécies, há poucos estudos sobre a resposta imunológica e o potencial alergênico das larvas para seres humanos. Com as larvas dos parasitos do gênero Hysterothylacium, foram realizados somente estudos experimentais, com o objetivo de avaliar reatividade cruzada com antígenos de Anisakis simplex (Caldas et al. 1998). Este trabalho tem por objetivo a investigação experimental sobre o potencial alergênico de larvas de terceiro estágio do parasito Hysterothylacium deardorffoverstreetorum com utilização de modelo murino.

\section{MATERIAL E METODOS}

No presente estudo, os parasitos foram coletados de peixes das espécies Cynoscion guatucupa (pescada maria-mole); Priacanthus arenatus (olho-de-cão); Paralichthys iscoceles (linguado), comercializados nos mercados de Niterói e Rio de Janeiro, no período de agosto de 2012 a novembro de 2014. As larvas foram, então, identificadas em microscópio óptico, seguindo as descrições realizadas por Knoff et al. (2012). Depois de identificados, os parasitos foram processados com três preparações diferentes: extrato bruto de larvas de Hysterothylacium deardorffoverstreetorum $(\mathrm{EBH})$, extrato secretado/excretado de larvas de Hysterothylacium deardorffoverstreetorum (ESH) e extrato bruto de larvas após excreção/secreção - esgotado - (EEH). O CEH foi obtido por meio da maceração do parasito íntegro. Já o ESH foi obtido mediante imersão de larvas vivas em meio ácido, a fim de induzir a excreção dos antígenos desejados. Já o EEH foi obtido por meio das larvas que passaram pelo processo de extração do antígeno excretado/secretado mediante a incubação de larvas L3 vivas em meio ácido, com uma concentração de ácido clorídrico entre 50 e $100 \mathrm{mMol} / \mathrm{L}$ em temperatura de $37^{\circ} \mathrm{C}$, segundo protocolo citado por Valls et al. (2003). Os antígenos foram obtidos conforme protocolo descrito por Perteguer et al. (1996). Em um homogeneizador de Potter (Thomas Phila USA), as larvas, em solução de cloreto de sódio a $0,9 \%$, foram desintegradas na presença de fluoreto fenilmetilsulfonil (PMSF). Posteriormente, foi centrifugada a $8500 \times \mathrm{g}$, em temperatura de $4^{\circ} \mathrm{C}$, por um período de 30 minutos na centrífuga (Internacional portable refrigerated centrifuga Model PR). A quantificação proteica dos extratos foi realizada pelo método de Lowry et al. (1951); foi utilizada albumina sérica bovina (BSA) $1 \mathrm{mg} / \mathrm{mL}$ como padrão.

Para o experimento, utilizaram-se camundongos $\mathrm{BALB} / \mathrm{c}$ de oito a 10 semanas de idade, criados e mantidos no biotério local (Núcleo de Animais de Laboratório - NAL-UFF). Esses animais foram alojados em ambientes com exaustão de ar, em temperatura ambiente a $23-25^{\circ} \mathrm{C}$, foram alimentados com Nuvilab CR-1 Chow (Nuvital Nutrientes S/A) e receberam água destilada tratada com $0,1 \%$ HCL ad libitum. Todos os procedimentos foram aprovados pelo Comitê de Ética da Universidade Federal Fluminense, sob o número 00137/09.

Foram utilizados três diferentes grupos, cada um com seis camundongos. Os animais foram imunizados pela via intraperitoneal com $10 \mu \mathrm{g}$ de cada preparação antigênica, associados a $2 \mathrm{mg}$ de hidróxido de alumínio, no volume de $0,2 \mathrm{~mL}$, nos dias zero, 21 e 42 do experimento. Como 
controle, os animais receberam, pela mesma via de inoculação, $2 \mathrm{mg}$ de hidróxido de alumínio. A contenção química para inoculação e sangria foi realizada com xilazina $200 \mu \mathrm{g} / \mathrm{kg}$, associada à ketamina $10 \mathrm{mg} / \mathrm{kg}$.

Obtenção e preparo do soro - Amostras de sangue foram colhidas do plexo retro-orbitário em um volume de $0,1 \mathrm{~mL}$ nos dias zero (controle antes da imunização), $14^{\circ}, 21^{\circ}, 28^{\circ}, 35^{\circ}, 42^{\circ}, 49^{\circ}$, $56^{\circ}$ e $70^{\circ}$ do experimento. $\mathrm{O}$ material foi inicialmente diluído 1:10 em salina fisiológica, centrifugado a 800 x g por 10 minutos, e, então, o soro foi separado.

A presença de anticorpos dos isotipos $\operatorname{IgE}$ e IgG, anti-Hysterothylacium nos soros dos camundongos foi determinada por meio do método imunoenzimático ELISA. Em placas com poços de fundo chato (Maxi - Sorp - Nunc), foram colocados antígenos de Hysterothylacium deardorffoverstreetorum separados segundo o método descrito anteriormente. $\mathrm{O}$ antígeno foi colocado na concentração de $20 \mu \mathrm{g}$ proteína $/ \mathrm{mL}$ em tampão contendo bicarbonato 0.05M, pH 9.6. As placas foram incubadas durante duas horas a $37^{\circ} \mathrm{C}$ e foram lavadas em tampão fosfato salina (PBS) $\mathrm{pH}$ 7.2. Os sítios livres foram bloqueados com solução de gelatina a 1\% em PBS (PBS-G) durante duas horas à temperatura ambiente. Em seguida, as placas foram lavadas três vezes em PBS contendo $0,05 \%$ de Tween 20 (PBS- T). Os soros foram diluídos em PBS-G de forma seriada na base 3 a partir de 1:40 e incubados por duas horas a $37^{\circ} \mathrm{C}$. Os anticorpos conjugados a peroxidase: anti-IgE (cadeia $\varepsilon$ ), (RatAnti-Mouse IgE - Invitrogen) e anti- IgG total (L e H) (1:10000), (RabbitAnti-MouseIgG, wholemolecule - Sigma), diluídos na solução de bloqueio, foram acrescidos à reação $(50 \mu \mathrm{L}$ /poço $)$ e as placas foram mantidas a $37^{\circ} \mathrm{C}$ durante uma hora. Após lavagem das placas com PBS-T, a reação com o substrato e o cromógeno se fez pela adição de $50 \mu \mathrm{L} /$ poço de solução coletada $10 \mu \mathrm{L}$ de peróxido de hidrogênio a $30 \%$ diluídos em $25 \mathrm{~mL}$ de tampão citrato a $0,1 \mathrm{M}$ em presença de $10 \mathrm{mg}$ de OPD (ortofenilenodiamina). As placas foram incubadas durante cinco minutos à temperatura ambiente. A reação enzimática foi interrompida com adição de solução de ácido sulfúrico $4 \mathrm{~N}$. A leitura de densidade óptica (DO) foi realizada na leitora de microplaca (Thermo Plate TP-READER) em comprimento de onda de $492 \mathrm{~nm}$. O resultado foi expresso por meio das médias aritméticas do somatório das DO das diluições. A análise dos resultados foi realizada pela comparação do somatório das DO de cada soro.

Grupos de camundongos BALB/c, imunizados com antígenos secretados e somáticos HD, receberam, na oitava semana após a imunização secundária, uma injeção intradérmica no pavilhão auricular de $20 \mu \mathrm{L}$ da solução antígeno somático com salina fisiológica a $1 \mathrm{mg} / \mathrm{mL}$. A espessura do pavilhão auricular foi feita antes da inoculação e 24, 48 e 72 horas após a injeção com micrômetro de mostrador (Mitutoyo $\mathrm{n}^{\mathrm{o}}$ 7301).

A análise estatística foi realizada por análise de variância com pós-teste de Tukey com o programa GraphPadInStat. $\mathrm{Na}$ análise estatística dos dados experimentais, foram considerados os valores significativos a partir de $\mathrm{P}<0,05$ (Rodrigues, 1996).

\section{RESULTADOS}

Os resultados obtidos no presente estudo demonstram que, após a primeira imunização nos três grupos experimentais, não houve elevação dos níveis de anticorpos IgG. No entanto, após a segunda e a terceira imunização (realizadas nos dias 21 e 42, respectivamente), houve uma gradativa elevação dos níveis de anticorpos até o $49^{\circ}$ dia e esses níveis foram estatisticamente significativos em comparação com as amostras séricas obtidas após a primeira imunização, conforme demonstrado na Fig. 1. Ao se analisarem os níveis de anticorpos produzidos ao longo do experimento, nenhuma diferença significativa foi observada entre os três grupos experimentais (EBH, ESH e EEH). Na Fig. 2, verifica-se que os grupos imunizados com EBH e ESH responderam, após a segunda imunização, no $35^{\circ}$ dia, com uma discreta, porém significativa, elevação dos níveis de $\operatorname{IgE}$ $(\mathrm{P}<0,05)$. Entretanto, a partir do $42^{\circ}$ dia da terceira imunização realizada, os três grupos responderam com uma marcante elevação de anticorpos $\operatorname{IgE}$ específicos, atingindo o nível 
máximo no $56^{\circ}$ dia, seguido de uma diminuição no $70^{\circ}$ dia. Diferentemente dos níveis de $\mathrm{IgG}$, houve diferença estatística nas outras preparações nos dias 14, 28 e 35 entre o EEH, que ficou um pouco abaixo dos demais antígenos. A avaliação da imunidade celular (Fig. 3) mensurada pela intradermorreação revelou que as três preparações antigênicas apresentaram um aumento do espessamento auricular nos três tempos avaliados $(24,48$ e 72 horas). No entanto, verificou-se, no tempo de 48 para 72 horas, que o grupo sensibilizado com EBH aumentou, diferentemente dos grupos ESH e EEH, que se mantiveram no mesmo patamar da leitura anterior. Os resultados obtidos indicam que as preparações antigênicas do HD apresentaram potencial imunogênico desencadeante da produção de anticorpos IgG e IgE específicos em camundongos BALB/c após a segunda imunização. Os animais imunizados apresentaram uma positividade na intradermorreação, principalmente para EBH, sugerindo um forte envolvimento da imunidade celular no processo imunológico estudado.

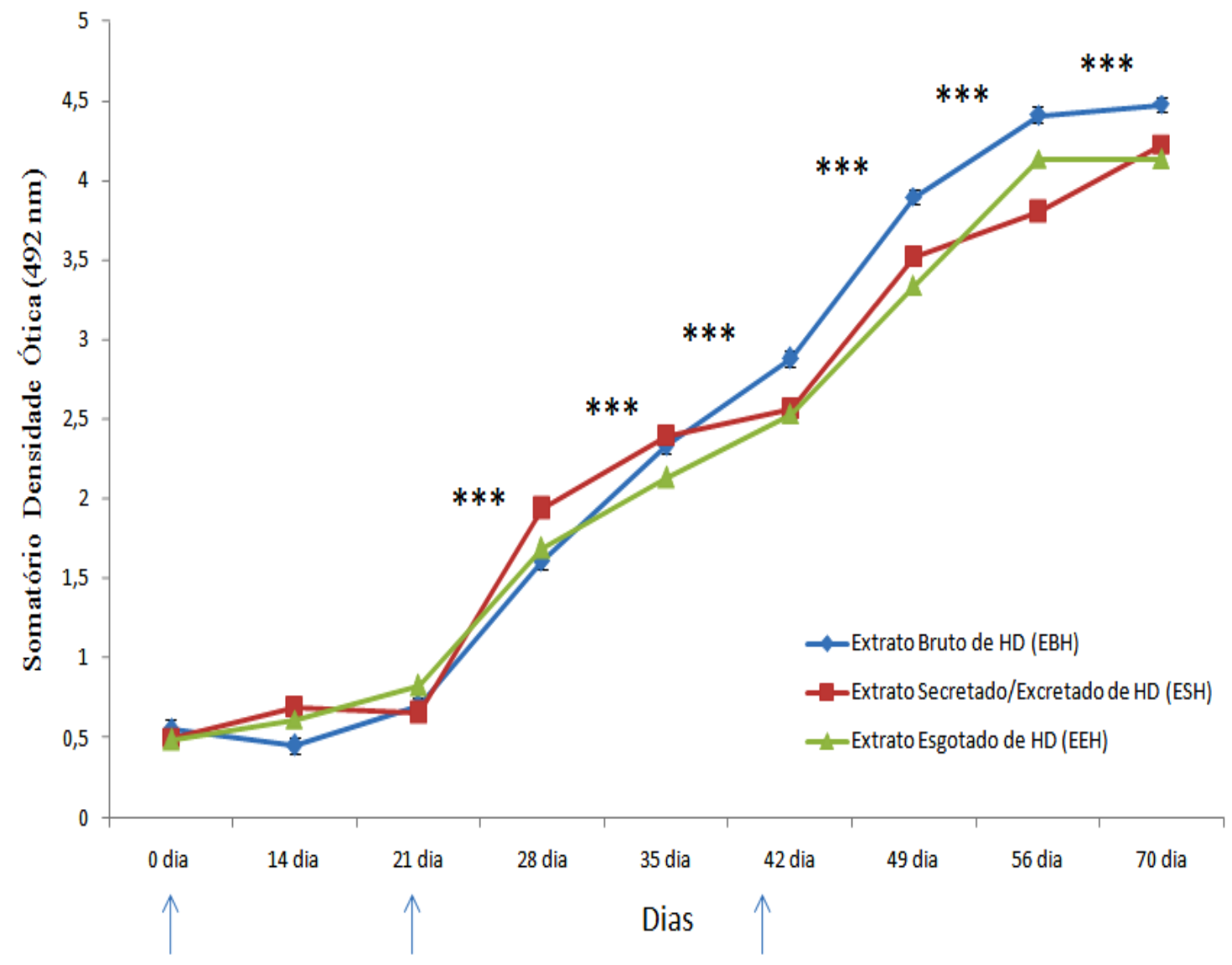

Figura 1. Níveis de anticorpos IgG anti-larvas de Hysterothylacium deardorffoverestreetorum. Camundongos foram imunizados com diferentes preparações antigênicas de larvas de $H$. deardorffoverestreetorum. As setas indicam as imunizações (zero, 21 e 42 dias). Os valores indicam as médias do somatório das $\mathrm{DO} \pm$ erro-padrão da média de cada grupo. A análise estatística foi realizada por meio de ANOVA, com *** $\mathrm{P}>0,001$ comparado ao dia zero. 


\section{Ribeiro et al.}

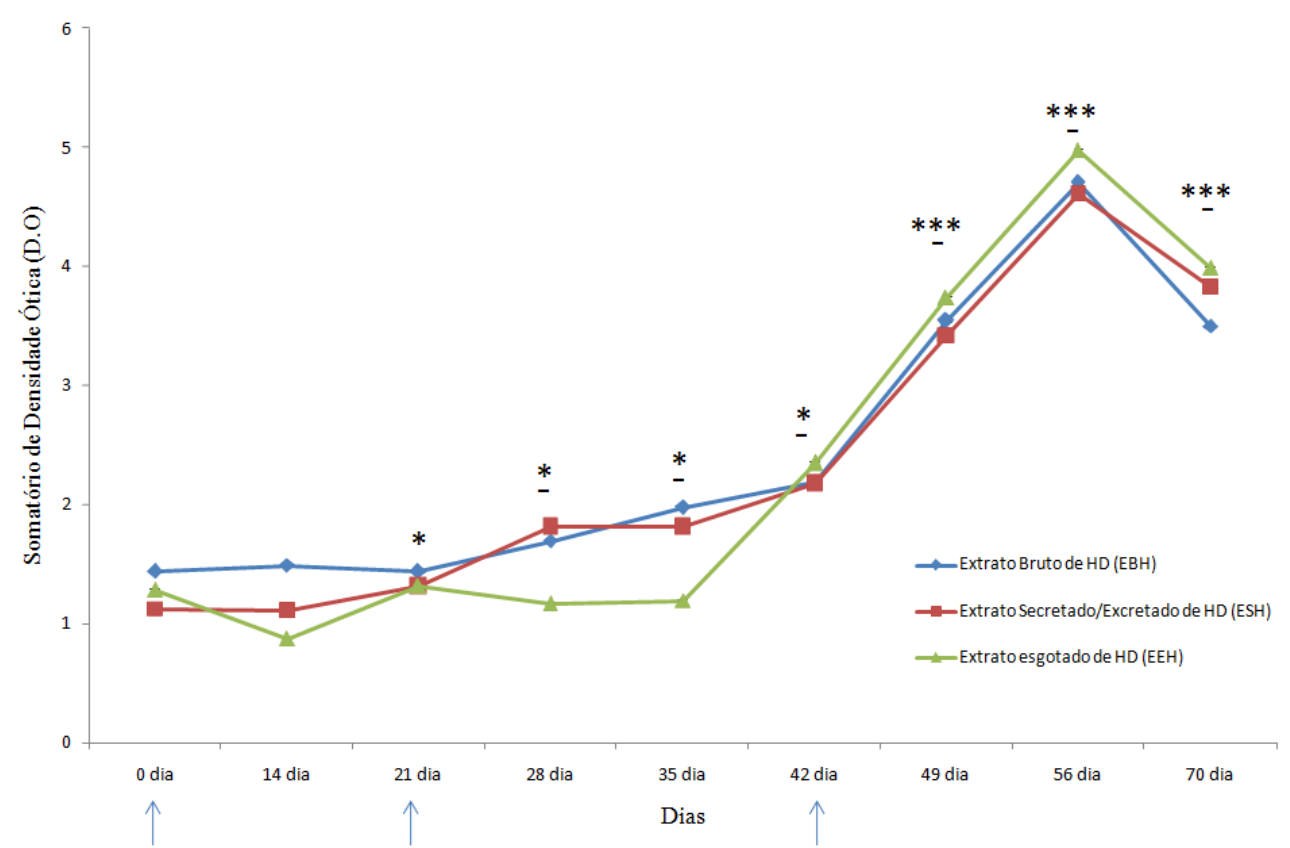

Figura 2. Níveis de anticorpos IgE anti-larvas de Hysterothylacium deardorffoverestreetorum. Camundongos foram imunizados com diferentes preparações antigênicas de larvas de $\mathrm{H}$. deardorffoverestreetorum. As setas indicam as imunizações (zero, 21 e 42 dias). Os valores indicam as médias do somatório das $\mathrm{DO} \pm$ erro-padrão da média de cada grupo. A análise estatística foi realizada por meio de ANOVA, com *P $>0,05$ e *** $\mathrm{P}>0,001$ comparado ao dia zero.

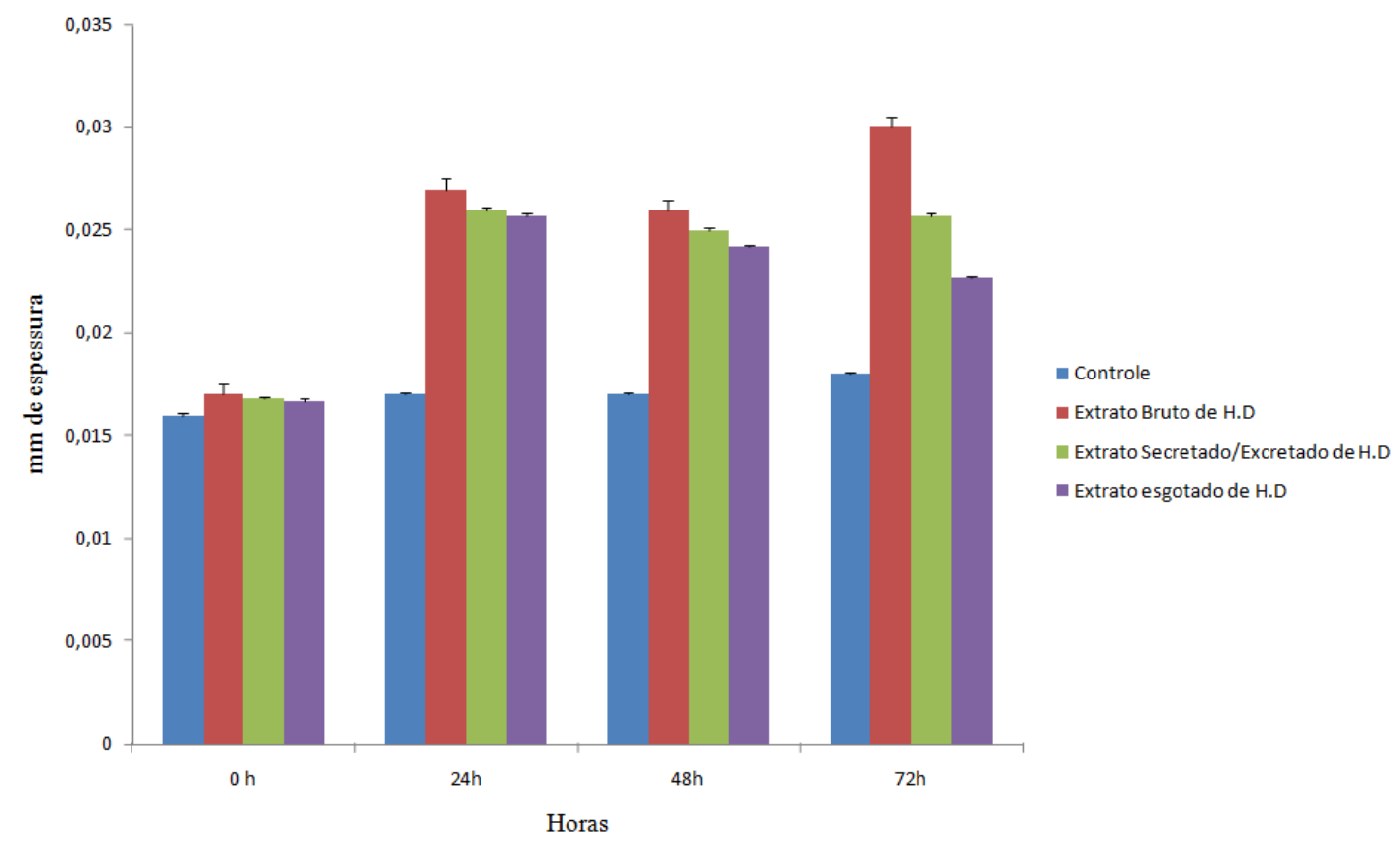

Figura 3. Mensuração da espessura do pavilhão auricular dos camundongos BALB/c sensibilizados com antígenos EBH, ESH e EEH. Camundongos foram imunizados com diferentes preparações antigênicas de larvas de $H$. deardorffoverestreetorum. As setas indicam as imunizações (zero, 21 e 42 dias). Os resultados estão expressos em $\mathrm{mm}$ de espessura \pm erro-padrão da média de cada grupo.A análise estatística foi realizada por meio de ANOVA, com $* \mathrm{P}<0,05 \mathrm{e}^{* * *} \mathrm{P}<0,001$ comparado à hora zero. 


\section{DISCUSSÃO}

Nas infecções por helmintos, a produção de $\operatorname{IgE}$ é um marco da resposta imunológica. Esta é consequência da ativação de um perfil linfocitário Th2 pelos helmintos, na qual predomina a secreção de IL-4 que, por sua vez, promove a mudança de classe de imunoglobulinas secretadas pelos linfócitos B para IgE. Apesar de este ser um conhecimento de longa data, a investigação inicial da resposta imunológica a larvas de terceiro estágio (L3) de A. simplex foi conduzida por meio da identificação da produção de anticorpos IgG específicos, mais abundantes no soro e, portanto, mais acessíveis à pesquisa (Cho e Lee, 2006). Embora controversa, a produção de $\operatorname{IgG} 4$ específica tem sido utilizada por investigadores para avaliação de doenças alérgicas, uma vez que esta imunoglobulina também se liga a epítopos reconhecidos pela $\operatorname{IgE}$ específica, sendo assim considerada como indicadora de estados de doença alérgica (Cho e Lee, 2006). Na forma alérgica de anisaquidose, o achado mais relevante é o aumento dos níveis de IgE total e específica. Em humanos, as respostas contra antígenos de $A$. simplex, tanto com anticorpos $\operatorname{IgE}$ quanto com IgG, são altamente heterogêneas, variando muito entre os indivíduos tanto quantitativa quanto qualitativamente (Audicana e Kennedy, 2008). Essa heterogeneidade de resposta pode ser observada em modelo experimental murino, em que a imunização com extrato bruto de larvas L3 de $A$. simplex na cavidade peritoneal resulta na maior produção de anticorpos específicos da classe IgG2a do que $\operatorname{IgG} 1$, o que indica um predomínio da resposta imunológica com padrão Th1 sobre a resposta Th2 (Cho e Lee, 2006). Em estudo realizado por Baeza et al. (2004) com o objetivo de avaliar as diferentes preparações antigênicas do A. simplex, comprovou-se que o produto proveniente da secreção/excreção do parasito mostrou-se mais alergênico que a preparação com antígeno somático. Esse resultado difere parcialmente do presente estudo, uma vez que, em relação ao HD, não houve diferença entre as preparações antigênicas na produção de IgG. Nos níveis avaliados de $\operatorname{IgE}$, houve diferença apenas no grupo imunizado com larvas após excreção (EEH), cujo resultado é abaixo dos demais antígenos, sugerindo que o antígeno mais alergênico está presente na secreção/excreção da larva ou na larva antes do tratamento em meio ácido, pois, na avaliação da imunidade celular, o antígeno bruto obteve resultado mais expressivo que os demais. Estudos alergênicos realizados anteriormente com larvas de Hysterothylacium spp. apontam o objetivo de avaliação de reatividade com outros nematoides, sobretudo com larvas de Anisakis sp. Em avaliação da reação cruzada testando-se soro de pacientes com anisaquidose alérgica com antígenos de $H$. aduncum, evidenciou-se reação positiva, confirmando a reação cruzada entre esses dois nematoides. (Fernandez-Caldas et al., 1998 e Marañon et al. 1998). Em estudo realizado por Iglesias et al. (1996), com o objetivo de avaliar a reatividade cruzada de Anisakis simplex e outros nematoides, incluindo o Hysterothylacium aduncum, foram fracionados diferentes antígenos. Utilizaram-se antígenos totais, antígenos secretado-excretados, antígenos do pseudoceloma e antígenos cuticulares. Observouse uma reação cruzada moderada em relação aos antígenos somáticos de $A$. simplex e $H$. aduncum. Outro estudo que comparou a reatividade cruzada de Anisakis simplex com diferentes antígenos, incluindo duas espécies diferentes, o Hysterothylacium aduncum e o $H$. fabri, foi realizado por Lozano Maldonado et al. (2004). Evidenciou-se a reação cruzada em ambos os antígenos testados, tanto somáticos quanto secretados.

\section{CONCLUSÃO}

O presente estudo é o primeiro relato do potencial patogênico em mamíferos desse parasito que está amplamente distribuído no continente americano. Esses resultados mostram que essas larvas são capazes de ativar o sistema imunológico tanto celular quanto humoral. Estudos posteriores serão necessários para melhorar o esclarecimento sobre o mecanismo de ação do parasito, as frações antigênicas mais importantes e a reatividade cruzada.

\section{REFERÊNCIAS}

AUDICANA, M.T.; KENNEDY, M.W. Anisakis simplex: from obscure infectious worm to inducer of immune hypersensitivity. Clinic. Microbiol. Rev., v.21, p.360-379, 2008.

BAEZA RODRÍGUEZ, A.; MATHEU, V.; RUBIO, M. et al Characterization of allergens secreted by Anisakis simplex parasite: clinical relevance in comparison with somatic allergens. Clinic. Exp. Allergy, v.34, p.296-302, 2004. 
BARROS, L.A.; MORAES FILHO, J.; OLIVEIRA, R.L. Nematóides com potencial zoonótico em peixes com importância econômica provenientes do rio Cuiabá. Rev. Bras. Ciênc. Vet., v.13, p.55-57, 2006.

CHO, M.K.; PARK, M.K.; KANG, S.A. et al. Allergenicity of two Anisakis simplex allergens evaluated in vivo using an experimental mouse model. Exp. Parasitol.,v.146, p.71-77, 2014.

CHO, S.W.; LEE, H.N. Immune reactions and allergy in experimental anisakiasis. Korean $\mathrm{J}$. Parasitol., v.44, p.271-283, 2006.

CRUZ, A.R.; SOUTO, P.C.S.; FERRARI, C.K.B. et al. Endoscopic imaging of the first clinical case of Anisakidosis in Brazil. Sci. Parasitol., v.11, p.97-100, 2010.

FERNANDEZ-CALDAS， E.; QUIRCE， S.; MARAÑON, F.; GOMEZ, M.L.D. Allergenic cross-reactivity between third stage larvae of Hysterothylaciumaduncum and Anisakis simplex. J. Allergy Clin. Immunol., v.101, p.554$555,1998$.

GRAPHPADINSTAT for windows XP. Version 4.10. San Diego: GraphPad Software. Available in:<www.graphpad.com Copyright 1992-1998>. Accessed in abril/2015

HOCHBERG, N.S.; HAMER, D.H. Anisakidosis: perils of the deep. Clin. Infect. Dis.; v.51, p.806-812, 2010.

IGLESIAS, R.; LEIRO, J.; UBEIRA, F.M.; SANTAMARINA,M.T.; NAVARRETE, I.; SANMARTÍN, M.L. Antigenic cross-reactivity in mice between third-stage larvae of Anisakis simpex and other nematodes. Parasitol. Res v.82, p. $378-381,1996$.
KNOFF, M.; FELIZARDO, N.N.; IÑIGUEZ, A.M. et al. Genetic and morphological characterisation of a new species of the genus Hysterothylacium (Nematoda) from Paralichthys isosceles Jordan, 1890 (Pisces: Teleostei) of the Neotropical Region, state of Rio de Janeiro, Brazil (1). Mem. Inst. Oswaldo Cruz, v.107, p.186-193, 2012.

LOWRY, O.H.; ROSEBROUGH, N.J.; FARR, A.L.; RANDALL, R.J. Protein mensurement with the folin phenol reagent. J. Biol. Chem., V.193, p.265-275, 1951.

MARAÑON, F.; FERNÁNDEZ-CALDAS, E.; QUIRCE, S.; DÍEZ GÓMEZ, M.L.; G'JÓN BOTELHA, H.; LÓPEZ ROMÁN, R.; LETI, C.B.F. Cross-reactivity between third stage larvae of Hysterothylacium aduncum and Anisakis simplex. J. allergy clin. immunol. v.101, n.1, part 2, p. s203, 1998.

PISCAGLIA, A.C.; VENTURA, M.T.; LANDOLFO, G. et al. Chronic anisakidosis presenting with intestinal intussusception. Eur. Rev. Med. Pharmacol. Sci.. v.18, p.3916-3920, 2014.

SABATER, E.I.L.; SABATER, C.J.L. Riesgos para lasaludasociados al parasitismo del pescado por nematodos de los géneros Anisakis y Pseudoterranova. Food Sci. Technol. Int., v.6, p.183-195, 2000.

VALLS, A.L, PASCUA, C.Y, MARTÍN ESTEBAN M. [Anisakis and anisakiosis]. Allergol. Immunopathol., v.31, p.348-355, 2003.

YAGI, K.; NAGASAWA, K.; ISHIKURA. et al. Female worm Hysterothylaciumaduncumexcreted from human: a case report. Jpn. J. Parasitol., v.45, p.12-23, 1996. 